\title{
Adhesión al tratamiento con levetiracetam: estudio observacional retrospectivo no intervencionista
}

\author{
Pedro J. Serrano-Castro, José A. Mauri-Llerda, Alberto García, Rodrigo Rocamora, Antonio Pardo-Merino, \\ Pilar García-García
}

Introducción. La adhesión al tratamiento es un factor que está repercutiendo en la eficacia de los fármacos antiepilépticos. El levetiracetam es un principio activo cuya eficacia y seguridad están ampliamente demostradas y que está disponible en diferentes formulaciones orales (sobres, comprimidos y solución oral), pero la información sobre la adhesión/cumplimiento del tratamiento con estas formulaciones orales es escasa.

Objetivo. Conocer la adhesión al tratamiento con formulaciones de levetiracetam (granulado en sobre y comprimidos) en adultos y ancianos.

Pacientes y métodos. Estudio observacional retrospectivo no intervencionista. Durante los tres meses previos al estudio debía estar en tratamiento con levetiracetam (granulado en sobre o comprimidos), bien en monoterapia o en combinación. Se valoraron los tests de cumplimiento (cuestionario de Morisky-Green modificado), así como un cuestionario de satisfacción del investigador y del paciente/cuidador. Los datos se analizaron con el programa SPSS v. 21.0.

Resultados. Un total de 466 pacientes completaron el estudio. La edad media fue de 52,75 $\pm 19,17$ años, y la media en los mayores de 65 años fue de 72,79 $\pm 6,15$ años. Un 55,4\% eran hombres. El cumplimiento se relaciona con la variable 'formulación galénica' y con la edad $(p=0,031)$. El riesgo de incumplimiento es un $86,4 \%$ mayor entre los que toman comprimidos frente a granulado de levetiracetam (odds ratio: 1,864). Asimismo, el incumplimiento es mayor entre los pacientes mayores de 65 años. El incumplimiento se relaciona con la atribución de fallos de memoria.

Conclusiones. Los pacientes más mayores presentan más dificultades en el cumplimiento. El granulado de levetiracetam en sobres favorece el cumplimiento.

Palabras clave. Adhesión. Epilepsia. Formulaciones galénicas. Levetiracetam.

\section{Introducción}

La adhesión al tratamiento se define clásicamente como el grado de coincidencia del comportamiento de un paciente en relación con los medicamentos que ha de tomar, el seguimiento de una dieta o los cambios que ha de hacer en su estilo de vida, con las recomendaciones de los profesionales de la salud que le atienden [1]. De una manera más sencilla, la Organización Mundial de la Salud definió la adhesión al tratamiento como la magnitud con la que el paciente sigue las instrucciones médicas [2].

En los últimos tiempos, se ha atribuido cada vez más relevancia a la influencia que la adhesión terapéutica puede tener sobre la calidad de vida y también sobre la morbimortalidad en enfermedades crónicas. La no adhesión, además, es un problema que también afecta al profesional de la salud [3]. En este sentido, han sido identificados diversos factores que influyen en la adhesión al tratamiento: socioeconómicos, relacionados con el equipo o el sistema de asistencia sanitaria, con la enfermedad, con el tratamiento y con el paciente. Todos estos factores van a tener una implicación directa sobre la calidad de vida del paciente [4-6].

Es bien conocido que un 50-75\% de los pacientes crónicos no se adhiere a sus indicaciones médicas [7]. En el caso de la epilepsia, un análisis de la bibliografía muestra que, aunque existe gran heterogeneidad en los datos, con cifras de adhesión de un 20-80\% [8-10], los índices de no adhesión parecen similares a los de otras enfermedades crónicas [11, 12]. Y ello sucede a pesar de que la falta de adhesión en la epilepsia podría tener repercusiones particularmente importantes. No en vano, diversos estudios han demostrado que la falta de adhesión en la epilepsia se relaciona con un incremento del riesgo de padecer más crisis epilépticas en el futuro [13, 14], del número de asistencias en los servicios de urgencias por traumatismos relacionados con crisis y del riesgo de accidentes con vehículos de motor [15].

En los últimos 25 años, el arsenal terapéutico de fármacos antiepilépticos (FAE) disponible para su uso en la práctica clínica se ha incrementado de ma-
Complejo Hospitalario Torrecárdenas; Almería (P.J. Serrano-Castro). Hospital Clínico Universitario Lozano Blesa; Zaragoza (J.A. Mauri-Llerda). Hospital Central Universitario de Asturias; Oviedo, Asturias (A. García). Hospital del Mar; Barcelona (R. Rocamora). Facultad de Psicología; Universidad Autónoma de Madrid (A. Pardo-Merino). Departamento Científico-Médico; Juste SAQF; Madrid, España (P. García-García).

Correspondencia:

Dra. Pilar García García. Departamento Científico-Médico. Juste SAQF. Avda. San Pablo, 27. E-28823 Coslada (Madrid).

Fax:

+34917248205.

E-mail:

pigarcia@grupojuste.com

Conflictos de interés:

P.G.G. está contratada por el departamento científico de Juste SAQF. A.P.M. completó el análisis estadístico del estudio bajo contrato con Juste SAQF. El resto de autores declara que no tiene conflictos de interés que afecten a los objetivos y resultados del estudio.

Nota:

Trabajo presentado como póster durante el I Congreso de la Sociedad Española de Epilepsia (Madrid, 2014).

Aceptado tras revisión externa: 19.04.16.

Cómo citar este artículo:

Serrano-Castro PJ, Mauri-Llerda JA, García A, Rocamora R, PardoMerino A, García-García P. Adhesión al tratamiento con levetiracetam: estudio observacional retrospectivo no intervencionista. Rev Neurol 2016; 62: 481-6.

(c) 2016 Revista de Neurología 
nera exponencial. A pesar de ello, la historia natural de la epilepsia no parece haber cambiado de manera significativa [16]. Desconocemos la forma en la que el grado de adhesión terapéutica podría condicionar la verdadera eficacia de dichos FAE $[2,17-20]$ $y$, por otro lado, la manera en que las diferentes formulaciones galénicas podrían influir sobre la adhesión terapéutica.

El levetiracetam es un principio activo indicado como monoterapia en el tratamiento de las crisis de inicio parcial con o sin generalización secundaria en pacientes mayores de 16 años con un nuevo diagnóstico de epilepsia. Está indicado como terapia concomitante en el tratamiento de las crisis de inicio parcial con o sin generalización secundaria en adultos, niños y lactantes desde 1 mes de edad con epilepsia; en el tratamiento de las crisis mioclónicas en adultos y adolescentes mayores de 12 años con epilepsia mioclónica juvenil; y en el tratamiento de las crisis tonicoclónicas generalizadas primarias en adultos y adolescentes mayores de 12 años con epilepsia generalizada idiopática [21].

En el momento actual, se encuentran disponibles diferentes formulaciones orales de levetiracetam en el mercado: sobres, comprimidos y solución oral [21]. Las formulaciones galénicas varían en tamaño; en este sentido, los comprimidos de levetiracetam tiene un tamaño de $19 \mathrm{~mm}$, y el granulado, de $2 \mathrm{~mm}$ [22]. Sin embargo, la información sobre la adhesión/ cumplimiento del tratamiento con estas formulaciones orales es escasa $[22,23]$.

El objetivo principal de este trabajo ha sido conocer la adhesión al tratamiento con diferentes formulaciones de levetiracetam (granulado en sobre y comprimidos) en adultos y ancianos. Como objetivos secundarios se planteó conocer si existían diferencias en cuanto al cumplimiento entre monoterapia y combinación, identificar factores demográficos (sexo, nivel de estudios y grupos etarios) o patológicos relacionados con el cumplimiento, y sugerir medidas o pautas que ayuden a minimizar los posibles riesgos derivados del tratamiento.

\section{Pacientes y métodos}

Se realizó un estudio observacional retrospectivo no intervencionista multicéntrico. Los pacientes fueron seleccionados a través de un muestreo aleatorio consecutivo realizado sobre los pacientes atendidos por 41 neurólogos en unidades de epilepsia de España. Todos los pacientes tenían que haber estado bajo tratamiento al menos con un fármaco cuyo principio activo fuera el levetiracetam, granulado en so- bre o comprimidos, bien en monoterapia bien en combinación durante los tres meses previos al inicio del estudio. El estudio fue aprobado por el Comité Ético de Investigación Clínica de Aragón.

Se recogió una variable dependiente denominada 'cumplimiento', que se cuantificó a partir de una versión modificada del cuestionario de Morisky-Green [19,24,25]. La versión modificada de este cuestionario incluye dos cuestiones adicionales al cuestionario original relacionadas con el uso de un pastillero $\mathrm{u}$ otras estrategias para el recuerdo de las tomas. Los pacientes han respondido a las preguntas de ese cuestionario en una escala de tipo Likert de cinco puntos: 'nunca,' 'casi nunca,' 'a veces', 'casi siempre' y 'siempre'. Los pacientes que han respondido 'nunca' o 'casi nunca' a las preguntas 1,3 y 4, y 'siempre' o 'casi siempre' a la pregunta 2 , han sido clasificados como 'cumplidores. Los restantes pacientes han sido clasificados como 'no cumplidores'.

Las variables independientes fueron de índole demográfico -edad (en años), sexo, estado civil, nivel de estudios y situación laboral- o clínico - diagnóstico, tratamiento actual, tabaquismo, índice de masa corporal, presión arterial sistólica, presión arterial diastólica, frecuencia cardíaca, tipo de crisis y etiología de la epilepsia-.

También se administró un cuestionario de satisfacción dirigido tanto al personal médico como al paciente o los cuidadores habituales que indagó sobre las preferencias subjetivas según la formulación galénica (granulado o comprimidos) en aspectos como eficacia, comodidad, facilidad para la deglución y precio.

Para estudiar la relación entre variables categóricas hemos utilizado la prueba $\chi^{2}$ de Pearson. Para estudiar la relación entre variables cuantitativas hemos utilizado el coeficiente de correlación de Pearson. Todos los cálculos se han realizado con el programa SPSS v. 21.

\section{Resultados}

En el estudio se han incluido 466 pacientes, cuyas características sociodemográficas y clínicas se recogen en la tabla I. Un 73,6\% del total de la muestra fue considerado 'cumplidor' según los criterios establecidos en el apartado de pacientes y métodos. El 26,4\% restante fue 'no cumplidor'.

No hemos encontrado diferencias significativas en el cumplimiento en función del sexo $\left(\chi_{(1)}^{2}=0,08\right.$; $p=0,78)$, el estado civil $\left(\chi_{(5)}^{2}=1,45 ; p=0,081\right)$ o el nivel de estudios $\left(\chi_{(3)}^{2}=0,18 ; p=0,98\right)$. Tampoco hemos encontrado relación entre el cumplimiento 
y algunas variables clínicas, como el índice de masa corporal $\left(R_{X Y}=0,03 ; p=0,493\right)$, el tabaquismo $\left(\chi_{(2)}^{2}=\right.$ $2,61 ; p=0,272)$, la etiología de la epilepsia $\left(\chi_{(20)}^{2}=\right.$ 21,07; $p=0,392)$ o estar recibiendo monoterapia o tratamiento combinado $\left(\chi_{(1)}^{2}=0,11 ; p=0,74\right)$.

$\mathrm{Al}$ analizar la información relativa al objetivo principal del estudio, hemos encontrado que el cumplimiento se relaciona tanto con la variable forma farmacéutica (comprimidos frente a granulado) como con la variable edad. La tabla II contiene la información relativa a estas variables.

Por lo que se refiere a la relación entre cumplimiento y forma farmacéutica, si se analiza toda la muestra no existe evidencia de tal relación $\left(\chi_{(1)}^{2}=\right.$ $1,59 ; p=0,207)$; sin embargo, en el grupo de menores de 65 años, el porcentaje de no cumplidores es significativamente mayor entre quienes utilizan la forma farmacéutica 'comprimidos' que entre quienes utilizan la forma farmacéutica 'granulado' (27,7\% frente a $\left.17,1 \% ; \chi^{2}{ }_{(1)}=4,63 ; p=0,031\right)$. El valor de la odds ratio en este grupo de edad $(1,864)$ indica que el riesgo de incumplimiento es un $86,4 \%$ mayor entre quienes utilizan comprimidos que entre quienes utilizan granulado.

Este resultado es congruente con las preferencias de forma farmacéutica expresadas por médicos y pacientes: entre los médicos, el 53,4\% prefiere granulado, el $18,7 \%$ prefiere comprimidos y al $27,9 \%$ restante le resulta indiferente la forma farmacéutica; entre los pacientes, el 49,5\% prefiere granulado, el $26,8 \%$ prefiere comprimidos y al $23,7 \%$ restante le resulta indiferente la forma farmacéutica. No obstante, la satisfacción con el medicamento es muy alta con ambas formas: el $89,4 \%$ de los médicos se manifiesta satisfecho o muy satisfecho con el granulado y el $79,8 \%$ se manifiesta satisfecho o muy satisfecho con los comprimidos; y el 82,4\% de los pacientes se manifiesta satisfecho o muy satisfecho con el granulado y el $69,4 \%$ se manifiesta satisfecho o muy satisfecho con los comprimidos.

En lo relativo a la relación entre cumplimiento y edad, hemos encontrado que el porcentaje de incumplidores es significativamente mayor en el grupo de mayores de 65 años que en el grupo de menores de 65 años $\left(33,9 \%\right.$ frente a $21,6 \% ; \chi^{2}{ }_{(1)}=8,69 ; p=$ $0,003)$. El valor de la odds ratio $(1,865)$ indica que el riesgo de incumplimiento es un $86,5 \%$ mayor en el grupo de más edad.

También hemos encontrado que la edad se relaciona con la forma farmacéutica utilizada: el porcentaje de pacientes que utilizan granulado es significativamente menor en el grupo de menos de 65 años que en el de más de 65 años (58\% frente a 69,4\%; $\left.\chi_{(1)}^{2}=6,21 ; p=0,013\right)$.
Tabla I. Características sociodemográficas y clínicas de la muestra.

\begin{tabular}{|c|c|c|c|c|}
\hline & & $\begin{array}{l}\text { Toda la muestra } \\
\qquad(n=466)\end{array}$ & $\begin{array}{l}\text { Hasta } 65 \text { años } \\
(n=283 ; 60,7 \%)\end{array}$ & $\begin{array}{c}>65 \text { años } \\
(n=183 ; 39,3 \%)\end{array}$ \\
\hline Edad (años) & & $52,75 \pm 19,17$ & $40,56 \pm 13,23$ & $72,79 \pm 6,15$ \\
\hline \multirow{2}{*}{ Sexo } & Hombres & $246(55,4 \%)$ & $148(53,6 \%)$ & $98(58,3 \%)$ \\
\hline & Mujeres & $198(44,6 \%)$ & $128(46,4 \%)$ & $70(41,7 \%)$ \\
\hline \multirow{6}{*}{ Estado civil } & Solteros & $129(27,8 \%)$ & $111(39,4 \%)$ & $18(9,9 \%)$ \\
\hline & Divorciados & $25(5,4 \%)$ & $20(7,1 \%)$ & $5(2,7 \%)$ \\
\hline & Casados & $239(51,5 \%)$ & $130(46,1 \%)$ & $109(59,9 \%)$ \\
\hline & Viudos & $48(10,3 \%)$ & $2(0,7 \%)$ & $46(25,3 \%)$ \\
\hline & Con pareja estable & $21(4,5 \%)$ & $17(6 \%)$ & $4(2,2 \%)$ \\
\hline & Sin pareja estable & $2(0,4 \%)$ & $2(0,7 \%)$ & 0 \\
\hline \multirow{4}{*}{$\begin{array}{l}\text { Nivel de } \\
\text { estudios }\end{array}$} & Sin estudios & $33(7,1 \%)$ & $14(5 \%)$ & $19(10,4 \%)$ \\
\hline & Primarios & $182(39,2 \%)$ & $80(28,4 \%)$ & $102(56 \%)$ \\
\hline & Secundarios & $155(33,4 \%)$ & $115(40,8 \%)$ & $40(22 \%)$ \\
\hline & Universitarios & $94(20,3 \%)$ & $73(25,9 \%)$ & $21(11,5 \%)$ \\
\hline \multirow{7}{*}{$\begin{array}{l}\text { Situación } \\
\text { laboral }\end{array}$} & Estudiantes & $51(11,1 \%)$ & $48(17,2 \%)$ & $3(1,7 \%)$ \\
\hline & Labores domésticas & $70(15,2 \%)$ & $40(14,3 \%)$ & $30(16,6 \%)$ \\
\hline & Trabajadores activos & $126(27,4 \%)$ & $116(41,6 \%)$ & $10(5,5 \%)$ \\
\hline & Baja laboral & $10(2,2 \%)$ & $9(3,2 \%)$ & $1(0,6 \%)$ \\
\hline & En paro & $44(9,6 \%)$ & $39(14,0 \%)$ & $5(2,8 \%)$ \\
\hline & Invalidez & $20(4,3 \%)$ & $14(5 \%)$ & $6(3,3 \%)$ \\
\hline & Jubilados & $139(30,2 \%)$ & $13(4,7 \%)$ & $126(69,6 \%)$ \\
\hline \multicolumn{2}{|c|}{ Diagnóstico confirmado } & $418(86,4 \%)$ & $261(88,2 \%)$ & $157(83,5 \%)$ \\
\hline \multirow{2}{*}{$\begin{array}{l}\text { Tratamiento } \\
\text { actual }\end{array}$} & Monoterapia & $305(65,5 \%)$ & $168(59,4 \%)$ & $137(74,9 \%)$ \\
\hline & Combinación & $161(34,5 \%)$ & $115(40,6 \%)$ & $46(25,1 \%)$ \\
\hline \multirow{3}{*}{ Tabaquismo } & No fumadores & $297(63,7 \%)$ & $191(67,5 \%)$ & $106(57,9 \%)$ \\
\hline & Exfumadores & $114(24,5 \%)$ & $45(15,9 \%)$ & $69(37,7 \%)$ \\
\hline & Fumadores & $55(11,8 \%)$ & $47(16,6 \%)$ & $8(4,4 \%)$ \\
\hline \multicolumn{2}{|c|}{ Índice de masa corporal } & $25,23 \pm 3,21$ & $24,74 \pm 3,23$ & $26,02 \pm 3,02$ \\
\hline \multicolumn{2}{|c|}{ Presión arterial sistólica (mmHg) } & $129,73 \pm 15,21$ & $125,36 \pm 13,69$ & $136,73 \pm 14,92$ \\
\hline \multicolumn{2}{|c|}{ Presión arterial diastólica (mmHg) } & $75,62 \pm 10,67$ & $73,01 \pm 9,01$ & $79,80 \pm 12,00$ \\
\hline
\end{tabular}


Tabla I. Características sociodemográficas y clínicas de la muestra (cont.).

\begin{tabular}{|c|c|c|c|c|}
\hline & & $\begin{array}{l}\text { Toda la muestra } \\
\qquad(n=466)\end{array}$ & $\begin{array}{l}\text { Hasta } 65 \text { años } \\
(n=283 ; 60,7 \%)\end{array}$ & $\begin{array}{c}>65 \text { años } \\
(n=183 ; 39,3 \%)\end{array}$ \\
\hline \multicolumn{2}{|c|}{ Frecuencia cardíaca (latidos/min) } & $73,28 \pm 10,09$ & $71,8 \pm 9,39$ & $75,67 \pm 10,73$ \\
\hline \multirow{5}{*}{ Tipo de crisis } & Parciales simples & $96(14,5 \%)$ & $52(12,7 \%)$ & $44(17,5 \%)$ \\
\hline & Parciales complejas & $216(32,7 \%)$ & $135(33 \%)$ & $81(32,3 \%)$ \\
\hline & Parciales con gen. & $188(28,5 \%)$ & $104(25,4 \%)$ & $84(33,5 \%)$ \\
\hline & $\begin{array}{l}\text { Generalizadas } \\
\text { tonicoclónicas }\end{array}$ & $128(19,4 \%)$ & $94(23 \%)$ & $34(13,5 \%)$ \\
\hline & Otros tipos & $32(4,8 \%)$ & $24(5,9 \%)$ & $8(3,2 \%)$ \\
\hline \multirow{7}{*}{ Etiología } & $\begin{array}{l}\text { Idiopática gen. } \\
\text { no identificada }\end{array}$ & $55(11,8 \%)$ & $47(16,6 \%)$ & $8(4,4 \%)$ \\
\hline & $\begin{array}{l}\text { Esclerosis } \\
\text { temporal mesial }\end{array}$ & $18(3,9 \%)$ & $13(4,6 \%)$ & $5(2,7 \%)$ \\
\hline & Postraumática & $45(9,7 \%)$ & $27(9,5 \%)$ & $18(9,8 \%)$ \\
\hline & Vascular & $119(25,5 \%)$ & $25(8,8 \%)$ & $94(51,4 \%)$ \\
\hline & Tumoral & $17(3,6 \%)$ & $7(2,5 \%)$ & $10(5,5 \%)$ \\
\hline & Criptogénica & $150(32,2 \%)$ & $114(40,3 \%)$ & $36(19,7 \%)$ \\
\hline & Otras causas & 62 (13,3\%) & $50(17,7 \%)$ & $12(6,6 \%)$ \\
\hline
\end{tabular}

Tabla II. Comportamiento de la muestra en relación con el levetiracetam.

\begin{tabular}{llllc}
\hline & Forma farmacéutica & Cumplidor & No cumplidor & Total \\
\hline \multirow{3}{*}{ Hasta 65 años } & Comprimidos & $86(72,3 \%)$ & $33(27,7 \%)$ & $119(42,0 \%)$ \\
\cline { 2 - 5 } & Granulado & $136(82,9 \%)$ & $28(17,1 \%)$ & $164(58,0 \%)$ \\
\cline { 2 - 5 } & Total & $222(78,4 \%)$ & $61(21,6 \%)$ & $283(60,1 \%)$ \\
\hline \multirow{3}{*}{ Com años } & Granulado & $37(66,1 \%)$ & $19(33,9 \%)$ & $56(30,6 \%)$ \\
\cline { 2 - 5 } & Total & $84(66,1 \%)$ & $43(33,9 \%)$ & $127(69,4 \%)$ \\
\hline \multirow{2}{*}{ Comprimidos } & $121(66,1 \%)$ & $62(33,9 \%)$ & $183(39,9 \%)$ \\
\cline { 2 - 5 } & Granulado & $123(70,3 \%)$ & $52(29,7 \%)$ & $175(37,6 \%)$ \\
\cline { 2 - 5 } & Total & $220(75,6 \%)$ & $71(24,4 \%)$ & $291(62,4 \%)$ \\
\hline
\end{tabular}

No hemos encontrado evidencia de que el porcentaje de cumplidores entre quienes utilizan siempre o casi siempre 'pastillero al salir de casa' sea dis- tinto del porcentaje de cumplidores entre quienes no utilizan pastilleros nunca o casi nunca $(78,6 \%$ frente a $\left.77,5 \% ; \chi_{(1)}^{2}=0,06 ; p=0,809\right)$. Tampoco hemos encontrado evidencia de que el porcentaje de cumplidores entre quienes utilizan siempre o casi siempre 'alguna estrategia para recordar tomar la medicación' sea distinto del porcentaje de cumplidores entre quienes no utilizan nunca o casi nunca alguna estrategia para recordar $(73,8 \%$ frente a $80,6 \%$; $\left.\chi_{(1)}^{2}=2,22 ; p=0,136\right)$.

Finalmente, aunque en principio no habíamos mantenido hipótesis alguna en relación con la atribución que los pacientes hacen a su falta de adhesión, hemos hallado que el cumplimiento se relaciona con la atribución del incumplimiento a los fallos de memoria. En concreto, el porcentaje de cumplidores es significativamente menor entre quienes hacen ese tipo de atribución que entre quienes no la hacen $\left(56,3 \%\right.$ frente a $\left.78,1 \% ; \chi_{(1)}^{2}=18,36 ; p<0,001\right)$.

\section{Discusión}

Éste es el primer estudio de ámbito nacional que trata de investigar las características relacionadas con la adhesión en uno de los FAE de tercera generación más utilizados y reconocido como FAE de primera línea por la mayoría de las guías de práctica clínica actuales.

Nuestra tasa global de 'no cumplidores' $(26,4 \%)$ podría parecer inferior a la de la mayoría de los estudios previos de ámbito europeo. Esta diferencia puede atribuirse a diferencias en los criterios para la definición del cumplimiento o en la metodología utilizada para medir la adhesión [26,27].

La eclosión de FAE de tercera generación en los últimos 25 años ha supuesto un incremento significativo en las opciones terapéuticas farmacológicas. Estos FAE podrían haber mejorado la calidad de vida de los pacientes en función de su mejor comportamiento desde el punto de vista farmacológico y de efectos adversos [28,29], pero parece comprobado que han fallado en el objetivo primordial de mejorar el pronóstico global de la epilepsia [16,29].

Las razones de este fallo podrían relacionarse con la ausencia de verdaderas novedades en las estrategias globales de tratamiento de la epilepsia. En este sentido, los FAE de tercera generación siguen siendo moléculas dirigidas a prevenir la recurrencia de crisis y no a tratar los mecanismos básicos de la enfermedad. En otras palabras, seguimos sin disponer de verdaderos fármacos antiepileptógenos [30,31].

Pero también es cierto que determinados factores relacionados con la manera en que los FAE de 
tercera generación se utilizan en la práctica clínica podrían condicionar su eficacia. En este sentido, la adhesión terapéutica podría tener un papel central porque, como se ha comentado, está demostrado que la no adhesión se relaciona con un incremento en el riesgo de padecer crisis epilépticas, así como de las consecuencias de ellas derivadas $[14,15,32]$.

Aunque existen estudios que analizan cómo influyen factores dependientes del paciente (clínicos, psicosociales o demográficos) sobre la adhesión [19, 33-35] y otros que analizan factores dependientes del FAE, como el número de tomas diarias o la complejidad del tratamiento [36,37], muy pocos estudios tratan aspectos más específicos relacionados con el FAE, como su forma farmacéutica.

Nuestros resultados muestran que algunas de las variables analizadas pueden relacionarse con mejores parámetros de adhesión. En este sentido, tal vez nuestro principal hallazgo es la evidencia de que la forma farmacéutica 'granulado' disminuye en un $86,4 \%$ el riesgo de incumplimiento terapéutico en el grupo de personas menores de 65 años. Este hallazgo adquiere especial importancia, ya que es precisamente el grupo de adultos jóvenes el que ha demostrado peores tasas de adhesión en estudios previos (Tabla II) $[33,34,38]$. La mayor facilidad en el consumo de la forma 'granulado', ya que se toma directamente, podría relacionarse con este resultado. En esa dirección, un estudio previo de nuestro grupo demostró que las circunstancias que dificultaran la ingesta de los medicamentos o supusieran un cambio en la rutina del paciente podrían empeorar la adhesión [19]. Esta hipótesis se ve respaldada por el hecho de que las personas menores de 65 años se manifiestan más satisfechas con el 'granulado' que con los comprimidos. El grupo de personas mayores de 65 años es menos sensible a estos cambios de rutina.

Un segundo hallazgo de interés es el mayor grado de cumplimiento entre personas menores de 65 años, independientemente de otras variables. Este hallazgo entra en conflicto con resultados de estudios previos, que habían descrito la menor edad como factor de riesgo para la falta de adhesión [33, 39]. Bien es cierto que estos estudios identifican la etapa juvenil como la de mayor riesgo de no adhesión y no la etapa de adulto joven, que es la que mayoritariamente integra nuestro grupo de menor edad. Es posible que, en nuestro caso, el tipo de epilepsia condicione esta diferencia, pues se sabe que las epilepsias de inicio en la etapa adulta suelen ser menos frecuentemente refractarias que las del adulto joven, y también que la epilepsia no refractaria se asocia a una mejor adhesión [19].
Destacamos que no encontramos diferencias en función del sexo o variables clínicas. Tampoco existen diferencias significativas entre pacientes en tratamiento en monoterapia frente a politerapia. El buen perfil de efectos adversos del levetiracetam podría justificar este último hallazgo, ya que otro hecho reconocido es que el perfil de efectos adversos, sobre todo en régimen de politerapia, es un factor de riesgo para la menor adhesión [32].

La principal limitación metodológica de nuestro estudio consiste en su naturaleza de estudio observacional y retrospectivo, lo que hace que las conclusiones obtenidas no estén respaldadas por un alto nivel de evidencia. Sin embargo, consideramos que las conclusiones de los estudios basados en la práctica clínica nos permiten acercarnos de manera fiable a lo que ocurre en nuestro entorno clínico más cercano.

En conclusión, factores relacionados con las características del FAE pueden ser importantes determinantes del grado de adhesión terapéutica que habitualmente no han sido tenidos en cuenta en la literatura previa. En este sentido, nuestro estudio demuestra que las personas menores de 65 años tienen una mejor adhesión al levetiracetam en su forma 'granulado', de mayor facilidad de deglución y consumo frente a los comprimidos.

Podría ser que formas farmacéuticas que eliminen trabas en el consumo de los medicamentos consigan una mejor adhesión terapéutica.

Son necesarios más estudios en el futuro que profundicen en los factores relacionados con el FAE que determinen el grado de cumplimiento terapéutico.

\section{Bibliografía}

1. Sackett DL, Haynes RB, Gibson ES, Hackett BC, Taylor PW, Roberts RS. Randomised clinical trial of strategies for improving medication compliance in primary hypertension. Lancet 1975; 1: $1205-7$.

2. Sabaté E. Adherencia a los tratamientos a largo plazo. Pruebas para la acción. Geneva: Organización Mundial de la Salud; 2004. URL: http://www.paho.org/spanish/ad/dpc/nc/adherencialargo-plazo.pdf. [15.07.2015].

3. Hov I, Bjartnes M, Slørdal L, Spigset O. Are drugs taken as prescribed? Tidsskr Nor Laegeforen 2012; 132: 418-22.

4. Gomes M, Maia FH. Medication-taking behavior and drug self regulation in people with epilepsy. Arch Neuropsiquiatria 1998; 56: 714-9.

5. Eatock J, Baker GA. Managing patient adherence and quality of life in epilepsy. Neuropsychiatr Dis Treat 2007; 3: 117-31.

6. McNicholas F. To adhere or not, and what we can do to help. Eur Child Adolesc Psychiatry 2012; 21: 657-63.

7. Wertheimer Al, Santella TM. Medication compliance research: still so far to go. J Appl Res Clin Exp Ther 2003; 3: 254-61.

8. Leppik IE. How to get patients with epilepsy to take their medication. The problem of noncompliance. Postgrad Med 1990; 88: 253-6.

9. Garnett WR. Antiepileptic drug treatment: outcomes and adherence. Pharmacotherapy 2000; 20: 191-9. 
10. Lannon SL. Using a health promotion model to enhance medication compliance. J Neurosci Nurs 1997; 29: 170-8.

11. Kyngäs H. Compliance with health regimens of adolescents with epilepsy. Seizure 2000; 9: 598-604.

12. Asadi-Pooya AA. Drug compliance of children and adolescents with epilepsy. Seizure 2005; 14: 393-5.

13. Cramer JA, Glassman M, Rienzi V. The relationship between poor medication compliance and seizures. Epilepsy Behav 2002; 3: $338-42$.

14. Manjunath R, Davis KL, Candrilli SD, Ettinger AB. Association of antiepileptic drug nonadherence with risk of seizures in adults with epilepsy. Epilepsy Behav 2009; 14: 372-8.

15. Davis KL, Candrilli SD, Edin HM. Prevalence and cost of nonadherence with antiepileptic drugs in an adult managed care population. Epilepsia 2008; 49: 446-54.

16. Brodie MJ. Road to refractory epilepsy: the Glasgow story. Epilepsia 2013; 54 (Suppl 2): S5-8.

17. Cramer JA, Roy A, Burrell A, Fairchild CJ, Fuldeore MJ, Ollendorf DA, et al. Medication compliance and persistence: Terminology and definitions. Value Health 2008; 11: 44-7.

18. Trinka E. Ideal characteristics of an antiepileptic drug: how do these impact treatment decisions for individual patients? Acta Neurol Scand Suppl 2012; 194: 10-8.

19. Serrano-Castro PJ, Pozo-Muñoz C, Alonso-Morillejo E, Martos-Méndez MJ, Bretones-Nieto B. Factores vinculados a la adhesión al tratamiento en pacientes con epilepsia refractaria y no refractaria. Rev Neurol 2011; 53: 721-8.

20. Goodman MJ, Durkin M, Forlenza J, Ye X, Brixner D. Assessing adherence-based quality measures in epilepsy. Int J Quality Health Care 2012; 24: 293-300.

21. Agencia Española de Medicamentos y Productos Sanitarios. Fichas técnicas de levetiracetam. URL: http://www.aemps. gob.es. [30.09.2015].

22. Ries S, Reifschneider G, Scheer W. Levetiracetam minitablets improve compliance in patients with epilepsy. Psychopharmakotherapie 2012; 19: 260-4.

23. Overgaard AB, Højsted J, Hansen R, Møller-Sonnergaard J, Christrup LL. Patients' evaluation of shape, size and colour of solid dosage forms. Pharm World Sci 2001; 23: 185-8.

24. Morisky DE, Ang A, Krousel-Wood M, Ward HJ. Predictive validity of a medication adherence measure in an outpatient setting. J Clin Hypertens (Greenwich) 2008; 10: 348-54.
25. Morisky DE, Green LW, Levine DM. Concurrent and predictive validity of a self-reported measure of medication adherence. Med Care 986; 24: 67-74.

26. Jones RM, Butler JA, Thomas VA, Peveler RC, Prevett M. Adherence to treatment in patients with epilepsy: associations with seizure control and illness beliefs. Seizure 2006; 15: 504-8.

27. Dash D, Sebastian TM, Aggarwal M, Tripathi M. Impact of health education on drug adherence and self-care in people with epilepsy with low education. Epilepsy Behav 2015; 44: 213-7.

28. Sander JW. The use of antiepileptic drugs - principles and practice. Epilepsia 2004; 45 (Suppl 6): S28-34.

29. Löscher W, Schmidt D. Modern antiepileptic drug development has failed to deliver: ways out of the current dilemma. Epilepsia 2011; 52: 657-78.

30. Weaver DF. Epileptogenesis, ictogenesis and the design of future antiepileptic drugs. Can J Neurol Sci 2003; 30: 4-7.

31. Schmidt D. Is antiepileptogenesis a realistic goal in clinical trials? Concerns and new horizons. Epileptic Disord 2012; 14: 105-13.

32. Faught E. Adherence to antiepilepsy drug therapy. Epilepsy Behav 2012; 25: 297-302.

33. Buck D, Jacoby NN, Bakefit GUS, Chadwick DW. Factors influencing drug regimes compliance with antiepileptic. Seizure 1997; 6: 87-93.

34. Mitchell WG, Scheier LM, Baker SA. Adherence to treatment in children with epilepsy: who follows 'doctor's orders'? Epilepsia 2000; 41: 1616-25.

35. Pozo-Muñoz C, Alonso-Morillejo E, Martos-Mendez M, Bretones-Nieto B, Serrano-Castro P. Psychosocial factors involved in adherence to treatment in patients with epilepsy. Psychol Health 2011; 2: S199.

36. Cramer JA, Arrigo C, Geneviève VH, Bromifield E. Comparison between the QOLIE-31 and derived QOLIE-10 in a clinical trial of levetiracetam. Epilepsy Res 2000; 41: 29-38.

37. Claxton AJ, Cramer J, Pierce C. A systematic review of the associations between dose regimens and medication compliance. Clin Ther 2001; 23: 1296-310.

38. Enríquez-Cáceres M, Soto-Santillana M. Incumplimiento farmacológico en pacientes con epilepsia. Rev Neurol 2006; 42: 647-54.

39. Horne R, Weinman J. Patients' beliefs about prescribed medicines and their role in adherence to treatment in chronic physical illness. J Psychosom Res 1999; 47: 555-67.

\section{Treatment adherence with levetiracetam: a non-interventionist retrospective observation-based study}

Introduction. Treatment adherence is a factor that is affecting the effectiveness of antiepileptic drugs. Levetiracetam is a drug whose effectiveness and safety is well established and is available in different oral formulations (granulates in sachets, tablets, oral solution), but information on treatment adherence/compliance with these oral formulations is limited.

Aim. To determine treatment adherence with levetiracetam formulations (granulates in sachets, tablets) in adult and elderly people.

Patients and methods. Retrospective observational non-interventionist study. During the three months before the study patients should be treated with levetiracetam (granulates in sachets or tablets), either alone or in combination. Compliance tests (Green-Morisky modified test) as well as a satisfaction questionnaire investigator and patient/caregiver is assessed. Data were analyzed using SPSS v. 21.0 program.

Results. A total of 466 patients completed the study. The average age was $52.75 \pm 19.17$ years old and the average over 65 years of $72.79 \pm 6.15$. 55.4\% were men. Compliance is related to the variable 'pharmaceutical formulation' and with age $(p=0.031)$. The risk of non-compliance was $86.4 \%$ higher among those taking tablets versus granulates in sachets of levetiracetam (odds ratio: 1.864). Likewise, the non-compliance was greater among patients over 65 years. The noncompliance was related to attributing of memory failures.

Conclusions. Patients older are more difficult to compliance. The granulates levetiracetam in sachets improves compliance.

Key words. Adherence. Epilepsy. Galenic formulations. Levetiracetam. 